\title{
NEW REDSHIFTS OF RADIO SOURCES FROM THE S4 AND S5 SURVEYS
}

\author{
W. Xu, C. R. Lawrence, A. C. S. Readhead, and T. J. Pearson \\ Owens Valley Radio Observatory, 105-24 California Institute of Technology, Pasadena, California 91125 \\ Received 1993 February 18; revised 1994 April 11
}

\begin{abstract}
Redshifts are reported for 23 objects in the Caltech-Jodrell Bank VLBI survey.
\end{abstract}

\section{INTRODUCTION}

We are engaged in VLBI surveys of two complete samples of radio sources. The Pearson-Readhead (PR) survey (Pearson \& Readhead 1988) contains 65 sources in the S4 (Pauliny-Toth et al. 1978) and S5 (Kühr et al. 1981) surveys, with $S(5 \mathrm{GHz}) \geqslant 1.3 \mathrm{Jy}, \delta>35^{\circ}$ and $|b| \geqslant 10^{\circ}$. The Caltech-Jodrell Bank (CJ) survey ${ }^{1}$ (Xu et al. 1993; Wilkinson et al. 1993) includes 135 sources in the S4 and S5 surveys with $0.7 \mathrm{Jy} \leqslant S(5 \mathrm{GHz})<1.3 \mathrm{Jy}$ in the same sky region, which can be combined with the PR sample to form a flux-limited complete sample of 200 sources. $^{2}$ The CJ survey aims at mapping all objects strong enough for MK II VLBI at both 1.67 and $5 \mathrm{GHz}$. The combined PR and CJ sample will make possible a more detailed classification of AGNs and provide a sample which is sufficiently large to make interesting statistical tests by class.

When we began the CJ survey in early 1990, about onethird of the objects had unknown redshifts or optical identifications. We therefore began a project at the Palomar Observatory to determine as many of the missing optical identifications and redshifts as possible. Our observations are not yet complete; however, we have determined about half of the missing redshifts.

\section{OBSERVATIONS}

All of the spectra were taken with the Double Spectrograph on the Palomar 200 in. telescope. A 300 line/mm

\footnotetext{
${ }^{1}$ The Caltech-Jodrell Bank VLBI survey is in collaboration with P. N. Wilkinson and A. Polatidis of Nuffield Radio Astronomy Laboratories, University of Manchester, Jodrell Bank, U.K.

${ }^{2}$ Two sources in the S4 survey are not included in the CJ sample. One is a planetary nebula. The other is actually a quasar pair, each of which has a flux density less than $0.7 \mathrm{Jy}$.
}

grating was used in the blue and a 158 line $/ \mathrm{mm}$ grating in the red, providing resolution of $9 \AA$ in the blue and $18 \AA$ in the red, respectively. An $800 \times 800$ Texas Instruments $C C D$ detector was used in both the blue and red. The incoming beam was divided with a dichroic beamsplitter either at 4800 $\AA$, giving an overall wavelength coverage of 3200-9300 or at $5200 \AA$, giving an overall wavelength coverage of 3800-10000 $\AA$. The dates of observations and integration times are listed in Table 1.

The rms error in fitting $\mathrm{He}-\mathrm{Hg}-\mathrm{Ar}$ arc lines was typically about $0.3 \AA$ in the blue and $0.5 \AA$ in the red. The wavelength scale was shifted slightly based on the He comparison spectra obtained immediately after each object spectrum to compensate for flexure in the instrument. Correction for atmospheric absorption and absolute flux density calibration were based on observations of Oke \& Gunn (1983) standard stars.

\section{REDSHIFTS}

After continuum subtraction, the emission and absorption features were modeled with Gaussian or Lorentzian functions. No statistical criteria were used to evaluate the quality of the fit; rather the residuals were compared visually to noise in adjacent parts of the spectrum. A weighted-mean redshift was calculated for each source, with the weight of each line component given by the ratio of height to width. An uncertainty was calculated by a weighted average of the deviations of the line components from the mean redshift and a $0.5 \AA$ wavelength error, added in quadrature. The results are presented in Table 1, along with a list of the line components used in the calculation. Many of the objects have complex spectra as can be seen in the table. Additional features, both in absorption and emission, are seen in some of the objects, but were not useful in determining the redshift and are therefore not listed. The spectra and further analysis will be published when the project is complete. 
TABLE 1. Redshifts of Caltech-Jodrell Bank VLBI Survey Sources.

\begin{tabular}{|c|c|c|c|c|c|c|}
\hline Source & $\begin{array}{c}\text { RA } \\
(1950.0)\end{array}$ & $\begin{array}{c}\text { Dec } \\
(1950.0)\end{array}$ & $z$ & $\begin{array}{l}\text { Integration } \\
\text { Time(s) }\end{array}$ & $\begin{array}{l}\text { Observation } \\
\text { Date }\end{array}$ & Features used in redshift determination \\
\hline $0010+775$ & 00:10:22.31 & $77: 32: 06.4$ & $0.326 \pm 0.001$ & 3000 & $22 \operatorname{Jan} 1990$ & $\begin{array}{l}\mathrm{Mg} \text { II } \lambda 2798, \quad[\mathrm{O} \mathrm{II}] \lambda 3727, \quad[\mathrm{O} \mathrm{III}] \lambda \lambda 4959,5007, \quad \mathrm{H} \alpha, \\
{[\mathrm{S} \text { II] } \lambda \lambda 6717,6734 .}\end{array}$ \\
\hline $0022+390$ & $00: 22: 46.67$ & 39:02:59.0 & $1.946 \pm 0.002$ & 3000 & 31 Dec 1989 & $\begin{array}{l}\operatorname{Ly} \alpha, \text { O I } \lambda 1303, \operatorname{Si} \text { IV } / O \text { IV } \lambda \sim 1400, \text { C IV } \lambda 1549, \text { C III] } \lambda 1909 \text {, } \\
\operatorname{Mg} \text { II } \lambda 2798 .\end{array}$ \\
\hline $0218+357$ & 02:18:04.13 & $35: 42: 32.0$ & ${ }^{a}$ & 3000 & 31 Dec 1989 & Featureless spectrum. \\
\hline $0402+379$ & $04: 02: 29.88$ & $37: 55: 26.9$ & $0.055 \pm 0.001$ & 3000 & 31 Dec 1989 & $\begin{array}{l}{[\mathrm{O} \text { I] }[\mathrm{S} \text { III] } \lambda 6300, \mathrm{O} \mathrm{I}] \lambda 6364, \mathrm{H} \alpha,[\mathrm{N} \text { II }] \lambda 6549,[\mathrm{~N} \text { II }] \lambda 6583,} \\
{[\mathrm{S} \mathrm{II}] \lambda \lambda 6717,6734 .}\end{array}$ \\
\hline $0407+747$ & $04: 07: 04.6$ & $74: 43: 29$ & - & 3000 & 31 Dec 1989 & Featureless spectrum. \\
\hline $0620+389$ & $06: 20: 51.53$ & $38: 58: 27.3$ & $3.469 \pm 0.004$ & 3000 & $23 \mathrm{Jan} 1990$ & Ly $\alpha, N \vee \lambda 1240$, Si IV/O IV $\lambda \sim 1400, \mathrm{C}$ IV $\lambda 1549, \mathrm{C}$ III] $\lambda 1909$. \\
\hline $0707+689$ & $07: 07: 55.24$ & $68: 57: 12.7$ & $1.141 \pm 0.002$ & 3000 & 23 Jan 1990 & $\left.\mathrm{C}_{\mathrm{III}}\right] \lambda 1909, \mathrm{Mg}$ II $\lambda 2798,[\mathrm{O} I 1] \lambda 3727$. \\
\hline $0716+714$ & $07: 16: 13.03$ & $71: 26: 15.2$ & $\longrightarrow$ & 1500 & 24 Jan 1990 & Featureless spectrum. \\
\hline $0740+828$ & $07: 40: 33.18$ & $82: 49: 24.2$ & $1.991 \pm 0.001$ & 1180 & 22 Jan 1990 & $\begin{array}{l}\text { Ly } \alpha, N \vee \lambda 1240, \text { Si IV } / O \text { IV } \lambda \sim 1400, \text { C IV } \lambda 1549, \mathrm{C} \text { III] } \lambda 1909 \text {, } \\
\text { Mg II } \lambda 2798 .\end{array}$ \\
\hline $0805+410$ & $08: 05: 33.63$ & $41: 01: 33.1$ & $1.420 \pm 0.003^{b}$ & 3000 & 22 Jan 1990 & $\mathrm{C}$ IV $\lambda 1549, \mathrm{C}$ III] $\lambda 1909, \mathrm{Mg}$ II $\lambda 2798$. \\
\hline $1003+830$ & $10: 03: 25.84$ & $83: 04: 56.7$ & $0.322 \pm 0.001$ & 3000 & $23 \mathrm{Jan} 1990$ & $\begin{array}{l}\mathrm{Mg} \text { II } \lambda 2798, \quad[\mathrm{O} \text { III }] \lambda \lambda 4959, \quad 5007, \quad \mathrm{H} \alpha, \quad[\mathrm{N} \text { II }] \lambda 6549, \\
{[\mathrm{~N} \text { II] }] \text { 6583. }}\end{array}$ \\
\hline $1020+400$ & $10: 20: 14.59$ & $40: 03: 27.2$ & $1.254 \pm 0.002^{b}$ & 1500 & $23 \operatorname{Jan} 1990$ & $\mathrm{Si}$ IV/O IV $\lambda \sim 1400, \mathrm{C}_{\text {IV }} \lambda 1549, \mathrm{C}$ III] $\lambda 1909, \mathrm{Mg}$ II $\lambda 2798$ \\
\hline $1053+704$ & $10: 53: 27.72$ & $70: 27: 47.9$ & $2.492 \pm 0.001$ & 3000 & 23 Jan 1990 & $\operatorname{Ly} \alpha, \mathrm{N} \vee \lambda 1240, \mathrm{C}$ IV $\lambda 1549, \mathrm{C}$ III] $\lambda 1909$ \\
\hline $1053+815$ & $10: 53: 36.22$ & $81: 30: 35.6$ & $0.706 \pm 0.006^{h}$ & 3000 & 23 Jan 1990 & $\mathrm{C}_{\mathrm{III}]} \lambda 1909, \mathrm{Mg}$ II $\lambda 2798$ \\
\hline $1128+385$ & $11: 28: 12.52$ & $38: 31: 51.5$ & $1.733 \pm 0.003$ & 3000 & 23 Jan 1990 & $\begin{array}{l}\text { Ly } \alpha, N \vee \lambda 1240, \text { Si IV } / O \text { IV } \lambda \sim 1400, \text { C IV } \lambda 1549, C \text { III] } \lambda 1909 \text {, } \\
\text { Mg II } \lambda 2798 .\end{array}$ \\
\hline $1144+402$ & $11: 44: 21.02$ & $40: 15: 14.1$ & $1.088 \pm 0.001^{c}$ & 2400 & 24 Jan 1990 & $\mathrm{C}$ IV $\lambda 1549, \mathrm{C}$ III] $\lambda 1909, \mathrm{Mg} \operatorname{II} \lambda 2798, \mathrm{H} \gamma$. \\
\hline $1144+542$ & $11: 44: 04.58$ & $54: 13: 22.8$ & $2.201 \pm 0.007$ & 3000 & 24 Jan 1990 & $\begin{array}{l}\operatorname{Ly} \alpha, N v \lambda 1240, \operatorname{Si} \text { IV } / O \text { IV } \lambda \sim 1400, \mathrm{C} \text { IV } \lambda 1549, \mathrm{C} \text { III] } \lambda 1909, \\
\operatorname{Mg} \text { II } \lambda 2798 .\end{array}$ \\
\hline $1225+368$ & $12: 25: 30.77$ & $36: 51: 47.0$ & $1.973 \pm 0.002^{h}$ & 3000 & $24 \mathrm{Jan} 1990$ & $\operatorname{Ly} \alpha, \operatorname{CIV} \lambda 1549$. \\
\hline $1242+410$ & $12: 42: 26.40$ & 41:04:30.0 & $0.813 \pm 0.002$ & 3000 & 24 Jan 1990 & $\begin{array}{l}\mathrm{C} \text { III] } \lambda 1909, \quad \mathrm{Mg} \text { II } \lambda 2798, \quad \mathrm{H} \beta, \quad[\mathrm{O} \mathrm{III]} \lambda \lambda 4959,5007, \\
{[\mathrm{O} \text { II] } \lambda 3727, \mathrm{H} \zeta .}\end{array}$ \\
\hline $1333+459$ & 13:33:15.70 & $45: 57: 56.4$ & $2.449 \pm 0.001^{d}$ & 2000 & 22 Jan 1990 & $\begin{array}{l}\text { O vi } \lambda 1035, \text { Ly } \alpha, N \text { v } \lambda 1240, \text { Si Iv /O Iv } \lambda \sim 1400, \text { C IV } \lambda 1549, \\
\text { He II } \lambda 1640, \text { OIII } \lambda 1667, \text { C III] } \lambda 1909 .\end{array}$ \\
\hline $1342+663$ & $13: 42: 41.04$ & $66: 21: 13.1$ & $1.351 \pm 0.003$ & 3000 & $23 \operatorname{Jan} 1990$ & Si IV /O IV $\lambda \sim 1400, \mathrm{C}$ IV $\lambda 1549, \mathrm{C}$ III] $\lambda 1909, \mathrm{Mg}$ II $\lambda 2798$ \\
\hline $1347+539$ & $13: 47: 42.57$ & $53: 56: 08.4$ & $0.980 \pm 0.003^{e}$ & 1500 & 22 Jan 1990 & $\mathrm{C}$ III] $\lambda 1909, \mathrm{Mg}$ II $\lambda 2798,[\mathrm{Ne} \mathrm{v}] \lambda 3426,[\mathrm{O}$ II] $\lambda 3727, \mathrm{H} \delta, \mathrm{H} \gamma$ \\
\hline $1418+546$ & 14:18:06.19 & $54: 36: 58.0$ & ${ }^{f}$ & 3000 & $22 \mathrm{Jan} 1990$ & Featureless spectrum. \\
\hline $1557+708$ & $15: 57: 37.1$ & $70: 49: 45$ & $0.026 \pm 0.001$ & 900 & $22 \operatorname{Jan} 1990$ & $\begin{array}{l}\text { Cross-correlation with an averaged spectrum of } 3 \mathrm{C} \text { galaxies in } \\
\text { the Pearson-Readhead sample (Lawrence et al. 1994). }\end{array}$ \\
\hline $1738+476$ & $17: 38: 36.32$ & $47: 39: 28.6$ & ${ }^{i}$ & 1800 & 9 Aug 1991 & Featureless spectrum. \\
\hline $1842+681$ & $18: 42: 43.49$ & $68: 06: 19.6$ & $0.472 \pm 0.002^{e}$ & 1800 & 9 Aug 1991 & $\mathrm{Mg}$ II $\lambda 2798,[\mathrm{Ne} \mathrm{v}] \lambda \lambda 3341,3426, \mathrm{H} \beta,[\mathrm{O}$ III] $\lambda \lambda 4959,5007, \mathrm{H} \alpha$. \\
\hline $1926+611$ & $19: 26: 49.65$ & $61: 11: 20.7$ & ${ }^{g}$ & 1800 & 9 Aug 1991 & Featureless spectrum. \\
\hline $2010+723$ & $20: 10: 16.21$ & $72: 20: 20.7$ & $\ldots g$ & 3000 & 9 Aug 1991 & Featureless spectrum. \\
\hline $2207+374$ & $22: 07: 11.72$ & $37: 27: 33.0$ & $1.493 \pm 0.004^{b}$ & 3000 & 9 Aug 1991 & $\begin{array}{l}\text { CIV } \lambda 1549, \\
\text { Mg II } \lambda 2798 .\end{array}$ \\
\hline $2229+695$ & $22: 29: 11.65$ & $69: 31: 02.7$ & $\underline{g}$ & 3600 & 9 Aug 1991 & Featureless spectrum. \\
\hline $2255+416$ & $22: 55: 04.68$ & $41: 38: 13.2$ & $\square^{i}$ & 3000 & 24 Jan 1990 & Featureless spectrum. \\
\hline $2311+469$ & $23: 11: 28.87$ & $46: 55: 54.4$ & $0.742 \pm 0.001$ & 1500 & $23 \operatorname{Jan} 1990$ & 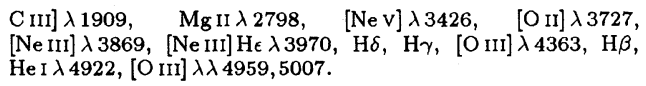 \\
\hline
\end{tabular}

Notes to Table 1

a O'Dea et al. (1992) reported a tentative redshift of 0.68 for the lens object. Carilli et al. (1993) confirmed it via HI absorption, and Browne et al. (1993) also confirmed it. However, the redshift of the quasar is still unknown.

$b$ Confirms redshifts suggested by Wills, Wills \& Douglas (unpublished).

c Confirms redshift reported by Vigotti et al. (1990).

${ }^{d}$ Confirms redshift published in the Veron-Cetty \& Veron catalog (5th edition)

e Confirms redshifts reported by Walsh et al. (1984).

$f$ Stickel et al. (1991) reported a redshift of 0.152 . Our observation cannot confirm their result.

$g$ There were thin clouds scattered on the sky during observation.

${ }^{h}$ Tentative redshift.

i A preliminary redshift from our work, referred by M. Stickel \& H. Kühr (1994, A\&AS, 103, 349), is not correct.

The reference search has made use of the NASA/IPAC Extragalactic Database (NED) which is operated by the Jet Propulsion Laboratory, California Institute of Technology, under contract with the National Aeronautics and
Space Administration. This work is supported in part by the National Science Foundation under Grants Nos. AST8814554 and AST-9117100 to the Owens Valley Radio Observatory. 


\section{REFERENCES}

Browne, I. W. A., Patnaik, A. R., Walsh, D., \& Wilkinson, P. N. 1993, MNRAS, 263, L32

'G' Carilli, C., Rupen, M., \& Yanny., B. 1993, in preparation

II. Kühr, H., Pauliny-Toth, I. I. K., Witzel, A., \& Schmidt, J. 1981, AJ, 86, 854

Lawrence, C. R., et al. 1994, ApJ (in press)

O'Dea, C. P., Baum, S. A., Stanghellini, C., Dey, A., van Breugel, W., Deustua, S., \& Smith, E. P. 1992, AJ, 104, 1320

Oke, J. B., \& Gunn, J. E. 1983, ApJ, 266, 713

Pauliny-Toth, I. I. K., Witzel, A., Preuss, E., Kühr, H., Kellermann, K. I., Fomalont, E. B., \& Davis, M. M. 1978, AJ, 83, 451

Pearson, T. J., \& Readhead, A. C. S. 1988, ApJ, 328, 114

Stickel, M., Padovani, P., Urry, C. M., Fried, J. W., \& Kühr, H. 1991, ApJ, 374,431
Veron-Cetty, M.-P., \& Veron, P. 1991, A Catalog of Quasars and Active Nuclei (5th Edition)

Vigotti, M., Merighi, R., Vettolani, G., Lahulla, J. F., \& Lopez-Arroyo, M' 1990, A\&AS, 83, 205

Walsh, D., Beckers, J. M., Carswell, R. F., \& Weymann, R. J.' 1984, MNRAS, 211, 105

Wilkinson, P. N., Polatidis, A. G., Readhead, A. C. S., Xu, W., \& Pearson, T. J. 1993, in Sub Arcsecond Radio Astronomy, edited by R. J. Davis and R. S. Booth, p. 213

Xu, W, Readhead, A. C. S, Pearson, T. J., Wilkinson, P. N., \& Polatidis, A. 1993, BAAS, 24, 1300 